East African Medical Journal Vol. 87 No. 1 January 2010

BONE MARROW EXAMINATION FINDINGS AT AGA KHAN UNIVERSITY HOSPITAL, NAIROBI

N.A. Okinda, MBChB, MMed, Clinical Pathologist and M. S. Riyat, MBChB, FRCPath (UK), Consultant HaematoOncologist, Department of Pathology, Aga Khan University Hospital, Nairobi, P.O. Box 30270-00100, Nairobi, Kenya

Request for reprints to: Dr. N.A. Okinda, Aga Khan University Hospital, Nairobi, P.O. Box 30270-00100, Nairobi, Kenya

\title{
BONE MARROW EXAMINATION FINDINGS AT AGA KHAN UNIVERSITY HOSPITAL, NAIROBI
}

\author{
N.A. OKINDA and M. S. RIYAT
}

\begin{abstract}
Objective: To establish the bone marrow examination findings and determine the indication for bone marrow examination.

Design: A retrospective audit.

Setting: Aga Khan University Hospital, Nairobi.

Subjects: All bone marrow aspirates done at Aga Khan University for the period comprising February 2003 to February 2006 were retrieved and analysed.

Results: A total of 356 patient's case histories including bone marrow examination results were analysed. Ages of the patients ranged from 18 months to 91 years. Males were $180(50.6 \%)$ and females were $176(49.4 \%)$. Nutritional anaemia as a group was the most common haematological disorder found on bone marrow examination in our patients with megaloblastic anaemia predominating. Acute myeloid leukaemia was the most common malignant haematological disorder. The most common indication for bone marrow examination was anaemia followed by diagnostic work up of fever of unknown origin.

Conclusion: Nutritional anaemia predominated as the commonest benign haematological finding on bone marrow examination while acute myeloid leukaemia was the most frequent haematological malignancy. Most bone marrow examinations were performed on patients with anaemia.
\end{abstract}

\section{INTRODUCTION}

Examination of the bone marrow is one of the most valuable diagnostic tests used to evaluate haematological disorders. Bone marrow aspiration and biopsy is also used to stage lymphoproliferative disorders and for prognostic purposes in chronic lymphoproliferative disorders such as chronic lymphocytic leukaemia.

Bone marrow aspiration and biopsy is also useful in the diagnostic workup of fever of unknown origin. It may reveal infections, such as tuberculosis, mycobacterium avium-intracellulare infections, histoplasmosis, leishmaniasis, and other disseminated fungal infections. It may be useful in establishing the diagnosis of storage diseases and diagnosing metastatic non-haemopoietic malignancies that havespread tothe bone marrow when all other imaging and diagnostic modalities have failed or when a leucoerythroblastic peripheral blood picture is present (1).

It is usually not indicated in haemolytic anaemia and is contraindicated in haemophilia and related congenital haemorrhagic disorders. Deviations from the normal may be qualitative with abnormal cellular morphology or quantitative with aplasia, hypoplasia or hyperplasia (2). Injury to the great vessels is a serious albeit rare complication of bone marrow sternal puncture.

The objective of this study was to establish the bone marrow examination findings and indications for bone marrow examination at Aga Khan University Hospital, Nairobi.

\section{MATERIALS AND METHODS}

This was a retrospective audit. All patients who underwent bone marrow aspiration during the period February, 2003 to February, 2006 were included in this study. Every patient's history was examined in detail and findings were recorded on a standard proforma including demographic data, symptoms and signs and all the relevant investigations. Bone marrow aspiration results were also recorded. Data was analysed to know the relative frequencies of different haematological disorders in our patients and also establish the indications for bone marrow examination. 


\section{RESULTS}

A total of 356 patient's case histories including bone marrow examination results wereanalysed. Ages of the patients ranged between 18 months to 91 years. Males were $180(50.6 \%)$ and females were $176(49.4 \%)$.

Theindication for the bone marrow examination were anaemia (26.9\%), bone marrow cultures $(17.5 \%)$, pancytopenia $(17.1 \%)$, confirmation of acute leukaemia or lymphoproliferative disorders $(10.9 \%)$, leukoerythroblastic picture $(7.5 \%)$, exclusion of multiple myeloma (8.4\%), staging of lymphomas $(6.5 \%)$, mono cytopenias $(5 \%)$ (Table 1$)$.
Acute myeloid leukaemia accounted for (8.1\%) and was found to be the most common malignant disorder, followed by low grade lymphoproliferative disorders - chronic lymphocytic leukaemia (6.7\%), multiple myeloma $(5.6 \%)$, acute lymphoblastic leukaemia $(5.6 \%)$, myeloproliferative disorders and myelodysplastic syndromes $(2 \%)$, bone marrow metastasis $(2.2 \%)$. Other miscellaneous findings included normal marrow (20.8\%), reactive marrow $(14.3 \%)$ and inadequacy of material for proper evaluation $(2.8 \%)$ (Table 3$)$.

Table 1

Indications for bone marrow examination

\begin{tabular}{|c|c|c|}
\hline \multirow[t]{2}{*}{ Indication } & \multicolumn{2}{|c|}{ Cases } \\
\hline & No & $(\%)$ \\
\hline Anaemia & 96 & 26.9 \\
\hline Pyrexia of unknown origin & 62 & 17.5 \\
\hline Pancytopenia & 61 & 17.1 \\
\hline Confirmed leukaemia (acute, chronic) & 36 & 10.9 \\
\hline Leucoerythroblastic picture & 27 & 7.5 \\
\hline Staging of lymphomas & 21 & 6.1 \\
\hline Mono cytopenias & 18 & 5 \\
\hline Indication for bone marrow examination not stated & 35 & 9 \\
\hline Total & 356 & \\
\hline
\end{tabular}

Nutritional anaemia contributed $14.9 \%$ amongst the non-malignant haematological disorders. Out of nutritional anaemia, megaloblastic anaemia was the most common i.e. ( $8.1 \%$ ) followed by iron deficiency anaemia $(6.5 \%)$ and mixed deficiencies $(0.3 \%)$.

Other non-malignant haematological disorders were aplastic anaemia $(1.3 \%)$, pure red cell aplasia $(0.6 \%)$, anaemia of chronic disease $(0.6 \%)$ idiopathic thrombocytopaenic purpura (ITP) $(4.2 \%)$, infections (fungal, tuberculosis, typhoid fever, malaria) $(3.7 \%)$ including HIV dyspoiesis (7.3\%) (Table 2).

Table 2

Spectrum of non-malignant haematological conditions

\begin{tabular}{llc}
\hline \multirow{2}{*}{ Disease } & \multicolumn{2}{l}{ Cases } \\
& No. & $(\%)$ \\
\hline Infections including HIV dyspoiesis & 39 & 11 \\
Megaloblastic anaemia & 29 & 8.1 \\
Iron deficiency anaemia & 23 & 6.5 \\
ITP & 15 & 4.7 \\
Aplastic anaemia & 5 & 1.3 \\
Suppression of bone marrow & & \\
by cytotoxics & 4 & 1.2 \\
Haemolytic anaemia & 4 & 1.2 \\
Anaemia of chronic disease & 2 & 0.6 \\
Pure red cell aplasia & 2 & 0.6 \\
Mixed deficiency & 1 & 0.3 \\
\hline Total & 124 & 35 \\
\hline
\end{tabular}

Table 3

Spectrum of malignant haematological conditions

\begin{tabular}{lll}
\hline Disease & \multicolumn{3}{l}{ Cases } \\
& No. & $(\%)$ \\
\hline Acute myeloid leukaemia & 29 & 8.6 \\
Lymphoproliferative disorders & 24 & 6.7 \\
Multiple myeloma & 20 & 5.6 \\
Myeloproliferative disorders / & & \\
myelodysplastic syndrome & 9 & 2 \\
Metastatic tumours to the & & \\
bone marrow & 9 & 2 \\
Acute lymphoblastic leukaemia & 6 & 1.7 \\
\hline Total & 97 & 26.6 \\
\hline
\end{tabular}

Haematinic deficiency was most common in young adults (19-40 years) (Table 4) so was aplastic anaemia and ITP; the mentioned disorders were also commoner in females. Haematological malignancies had a varied distribution with the myeloid leukaemia and lymphoproliferative disorders being commoner in young adults and myeloproliferative/myelodysplastic disorders occurring more in the older age group i.e. $>40$ years with a more preponderance for males (Table 5). Acute lymphoblastic leukaemia was commoner in the younger age group less the 18 years with an equal sex distribution. 
Table 4

Age distribution of bone marrow aspirate findings

\begin{tabular}{|c|c|c|c|c|}
\hline Bone marrow finding & $\begin{array}{c}1-18 \text { years } \\
\% \text { cases }\end{array}$ & $\begin{array}{c}19-40 \text { years } \\
\% \text { cases }\end{array}$ & $\begin{array}{c}>40 \text { years } \\
\% \text { cases }\end{array}$ & Total cases \\
\hline Infections including HIV dyspoiesis & 10 & 67 & 23 & 39 \\
\hline Megaloblastic anaemia & 21 & 52 & 27 & 29 \\
\hline Iron deficiency anaemia & 17 & 57 & 26 & 23 \\
\hline ITP & 67 & 33 & 15 & \\
\hline Aplastic anaemia & 0 & 100 & 0 & 5 \\
\hline Bone marrow suppression by drugs & 0 & 0 & 100 & 4 \\
\hline Haemolytic anaemia & 0 & 50 & 50 & 4 \\
\hline Anaemia of chronic diseases & 0 & 100 & 0 & 2 \\
\hline Pure red cell aplasia & 50 & 50 & 0 & 2 \\
\hline Mixed deficiency & 100 & 0 & 0 & 1 \\
\hline Acute myeloid leukaemia & 11 & 50 & 39 & 29 \\
\hline Lymphoproliferative disorders & 7 & 50 & 43 & 24 \\
\hline Multiple myeloma & 5 & 18 & 77 & 20 \\
\hline \multicolumn{5}{|l|}{ Myeloproliferative } \\
\hline disorders/myelodysplastic syndrome & 12 & 41 & 47 & 9 \\
\hline Metastatic tumour to the bone marrow & 0 & 67 & 33 & 9 \\
\hline Acute lymphoblastic leukaemia & 50 & 33 & 17 & 6 \\
\hline Normal marrow & 15 & 43 & 42 & 74 \\
\hline Reactive marrow & 7 & 46 & 47 & 51 \\
\hline Inadequate material for evaluation & 50 & 20 & 30 & 10 \\
\hline
\end{tabular}

ITP = Idiopathic thrombocytopaenic purpura

Table 5

Sex distribution of bone marrow aspirate findings

\begin{tabular}{lccc}
\hline Bone marrow finding & $\begin{array}{c}\text { Female } \\
\% \text { cases }\end{array}$ & $\begin{array}{c}\text { Male } \\
\% \text { cases }\end{array}$ & Total cases \\
\hline Infections including HIV dyspoiesis & 42 & 58 & 39 \\
Megaloblastic anaemia & 66 & 34 & 29 \\
Iron deficiency anaemia & 56 & 44 & 23 \\
ITP & 66 & 34 & 15 \\
Aplastic anaemia & 50 & 50 & 5 \\
Bone marrow suppression by drugs & 60 & 40 & 4 \\
Haemolytic anaemia & 75 & 25 & 4 \\
Anaemia of chronic diseases & 0 & 100 & 2 \\
Pure red cell aplasia & 50 & 50 & 2 \\
Mixed deficiency & 100 & 0 & 29 \\
Acute myeloid leukaemia & 42 & 58 & 24 \\
Lymphoproliferative disorders & 50 & 50 & 20 \\
Multiple myeloma & 59 & 41 & 9 \\
Myeloproliferative disorders/myelodysplastic syndrome & 47 & 53 & 9 \\
Metastatic tumour to the bone marrow & 33 & 67 & 6 \\
Acute lymphoblastic leukaemia & 50 & 50 & 74 \\
Normal marrow & 42 & 58 & 51 \\
Reactive marrow & 47 & 53 & 10 \\
Inadequate material for evaluation & 50 & 50 & \\
\hline ITP I & & & \\
\hline
\end{tabular}

ITP = Idiopathic thrombocytopenic purpura 


\section{DISCUSSION}

The spectrum of haematological disorders in patients is very wide. Bone marrow examination is a useful test in reaching the final diagnosis. It is one of the most common and relatively safe procedures done on patients in medical practice although injury to the great vessels may be a rare complication of bone marrow sternal puncture. Rarely have infection, excessive bleeding or embolism been reported after bone marrow biopsy (3).

This study shows that amongst the micronutrient anaemia, megaloblastic anaemia is the most common non-malignant disorder in our patients. In other similar studies its frequency ranges from as low as $24 \%$ to as high as $68 \%(4,5)$. In almost all these studies pancytopenia was the main presentation and so was the case in our study. Rarely megaloblastic anaemia may present with thrombocytopenia only (6).

The cause of megaloblastic anaemia is folate deficiency and vitamin B12 deficiency. The usual presenting feature of megaloblastic anaemia in our patients was macrocytic anaemia and symptoms of pancytopenia (thrombocytopenia and neutropenia).

Amongst other micronutrient anaemia, iron deficiency accounted for $6.5 \%$ only. This percentage is much lower than expected as an estimated 60 to $80 \%$ of the world's population has this nutritional deficiency (7). The possible explanation is that majority of the patients are diagnosed on peripheral blood smear examination and on serum ferritin levels and are treated as outpatients.

Aplastic anaemia was also reported in some of our patients $(1.3 \%)$. Epidemiologically, aplastic anaemia has a pattern of geographic variation opposite to that of leukaemia, with higher frequency in the developing world than in the industrialised West. Although not a common disease worldwide, aplastic anaemia has a social impact disproportionate to its incidence. Large prospective studies indicate an annual incidence of two new cases per million populations in Europe and Israel (8). The rate is much higher in the developing world, where aplastic anaemia may rival acute myelogenous leukaemia in frequency of diagnosis in haematology clinics. This has been shown from the studies in Thailand and China, where the incidence has been determined to be about threefold that in the West (9). European studies have confirmed and quantified medical drugs as risks for the development of marrow failure. Surprisingly, drug use accounts for only a small fraction of the disease in Thailand, where it is almost always idiopathic. Prognosis is directly related to the reduction in peripheral blood counts, particularly the neutrophil number: $<200$ granulocytes $/ \mu \mathrm{L}$ defines the category of severe disease (10). Recurrent bacterial sepsis or fungal invasion of critical organs secondary to refractory neutropenia is the usual cause of death. Its exact aetiology is still not known but an autoimmune mechanism has been inferred from positive responses to non transplant therapies and laboratory data (11).

Idiopathic thrombocytopaenia purpura was the third most common non malignant haematological disorder found on bone marrow examination in our patients. Idiopathic thrombocytopaenic purpura (ITP) is a condition of having a low platelet count (thrombocytopenia) of no known cause (idiopathic). As most causes appear to be related to antibodies against platelets, it is also known as immune thrombocytopenic purpura. The incidence of ITP is 50-100 new cases per million per year, with children accounting for half of that amount. More than $70 \%$ of the cases in children end up in remission within six months whether treated or not. Moreover, a third of the remaining chronic cases remit during the followup observation, and another third ended up with only mild thrombocytopenia ( $>50,000$ platelets per $\mu \mathrm{L})$ (12). The paediatric age group in our study did not have a case of ITP and this can be attributed to the fact that bone marrow examination is hardly ever needed to make a diagnosis of paediatric ITP. ITP is usually chronic in adults (13) and the probability of durable remission is $20-40 \%$ (14). The male: female ratio in the adult group is 1:1.2-1.7 (for children it is $1: 1)$ and the median age of adults at the diagnosis is 56-60 (15). This was hardly the case in our study where the condition was rampant in the young adults (19-40 years).

In this study, $29(8.1 \%)$ cases of leukaemia were noted, with acute myeloid leukaemia being the commonest, which was followed by low grade lymphoproliferative disorders mainly chronic lymphocytic leukaemia. Acute myeloid leukaemia (AML), also known as acute myelogenous leukaemia, is a cancer of the myeloid line of leucocytes, characterised by the rapid proliferation of abnormal cells which accumulate in the bone marrow and interfere with the production of normal blood cells. AML is the most common acute leukaemia affecting adults, and itsincidence increases with age. Although AML is a relatively rare disease, accounting for approximately $1.2 \%$ of cancer deaths in the United States (16) its incidence is expected to increase as the population ages.

Normal and reactive bone marrows were found in one third of our patients and therefore a confirmatory diagnosis was made in two thirds of the patients. This goes to show that bone marrow examination is a useful test and diagnosis can be made in a majority of patients, where the procedure is indicated.

In about $2.8 \%$, the bone marrow aspirate material was inadequate for evaluation and this could point towards a fibrotic lesion in the bone marrow or 
inadequate technique. This procedure is fairly easy to perform and yields information in almost all bone marrow examination, as shown by the low incidences of getting inadequate material for evaluation.

In conclusion, amongst the non-malignant haematological disorders, nutritional anaemia as a group was the most common disorder found on bone marrow examination in this study, megaloblastic anaemia being the most common followed by iron deficiency anaemia. HIV dyspoiesis was the second commonest bone marrow finding in this study. Acute myeloid leukaemia was the most common haematological malignancy followed by lymphoproliferative disorders. The most common indication for bone marrow examination was anaemia followed by bone marrow cultures for diagnostic work up of fever of unknown origin.

\section{ACKNOWLEDGEMENTS}

To the staff Department of Haematology for help and cooperation and for providing data on bone marrow examinations and the medical records staff for the provision of patients' files.

\section{REFERENCES}

1. Dimitrios V. Bone marrow aspiration and biopsy, emedicine last updated in June 28, 2004.

2. Mwangi, J. Bone marrow aspirate cytologies. Medicom. 1999; 14: 15 - 19.

3. Onal, I.K., Sumer, H., Tufan, A. and Shorbagi, A. Bone marrow embolism after bone marrow aspiration and biopsy. Am J. Hematol. 2005; 78: 158.

4. Ng, S.C., Kuperan, P., Chan, K.S., Bosco, J. and Chan, G.L. Megaloblastic anaemia-a review from University Hospital, Kuala Lumpur. Ann. Acad. Med. Sing. 1988; 17: 261-266.
5. Modood-ul-Mannan, Anwar, M., Saleem, M., Wigar, A. and Ahmad, M.A. Study of serum vitamin B 12 and folate levels in patients of megaloblastic anaemia in northern Pakistan. J. Pak. Med. Assoc. 1995; 45: 187-188.

6. Mohammed, A. J. Thrombocytopenia in children. J. Postgr. Med. Inst. 2004; 18: 353-358.

7. Andrew, N.C. Medical progress: disorders of iron metabolism. N. Eng. J. Med. 1999; 341: 1986-1995.

8. Young, N.S. Hematopoietic cell destruction by immune mechanisms in acquired aplastic anaemia. Semin. Hematol. 2000: 37: 3-14.

9. Issaragrisil, S., Leaverton, P.E., Changsung, K., et al. The Aplastic Anaemia Study Group; The incidence of aplastic anaemia in Thailand. Am. J. Hematol. 1999; 61: 164-168.

10. Neal, S. Young, J., Abkowitz, L. and Lucio, L. New insights into the pathophysiology of acquired cytopenias. Haematology. 2000: 18-38.

11. Chongli, Y. and Xiaobo, Z. Incidence survey of aplastic anaemia in China. Chin. Med. Sci. J. 1991; 6: 203-207.

12. Watts, R.G. Idiopathic thrombocytopenic purpura: a 10-year natural history study at the children's hospital of alabama. Clinical Paed. 2004; 43: 691-702.

13. Cines, D.B. and Blanchette, V.S. Immune thrombocytopenic purpura. N. Engl. J. Med. 2002; 346: 995-1008.

14. Stevens, W., Koene, H., Zwaginga, J.J. and Vreugdenhil, G. Chronic idiopathic thrombocytopenic purpura: present strategy, guidelines and new insights. Netherlands J. Med. 2006; 64: 356-363.

15. Cines, D.B. and Bussel, J.B. How I treat idiopathic thrombocytopenic purpura (ITP). Blood. 2005; 106: 2244-2251. doi:I0.1182/blood-2004-12-4598.

16. Jemal, A., Thomas, A., Murray, T. and Thun, M. Cancer statistics, 2002. CA Cancer J. Clin. 2002; 52: 23-47. 